\title{
TRANSFORMED DATA RELAYING METHOD FOR COVERAGE HOLE PRONE WIRELESS SENSOR NETWORK
}

\author{
R.Rajesh \\ Research Scholar, Department of Information Technology, Annamalai University, \\ Chidambaram, Tamil Nadu- 608002, India \\ mtechrajeshr@gmail.com \\ Dr. V. Tamizhazhagan \\ Assistant Professor, Department of Information Technology, Annamalai University, \\ Chidambaram, Tamil Nadu- 608002, India
}

rvtamizh@gmail.com

\begin{abstract}
Wireless Sensor Network (WSN) is a collection of tiny sensing devices capable of aggregating and transmitting environmental information to common sink nodes. Due to limited battery capacity, the nodes cover a small region wherein multi-hop communications are adapted for long-distance relaying. Early energy drain and node replacement results in the coverage-hole problem, resulting in information loss. This paper introduces a Distance-to-Coverage Transformed Relaying (DCTR) method to lessen information loss due to connectivity issues. The proposed method relies upon a conclusive machine learning paradigm for analyzing the residual energy-to-path existence feature of a neighboring node. Based on the residual energy, the distance or coverage metric is satisfied for non-intervening transmissions. The learning outcome provides definite transmission intervals preventing additional delay due to re-discovery. The transmissions are confined in the provided interval recommended, based on connectivity and coverage. The performance of the proposed method is analyzed using coverage rate, data loss ratio, and energy consumption. The proposed method improves the coverage factor by $7.93 \%$, reduces data loss by $9.64 \%$, and energy consumed by $8.49 \%$ for different unconnected nodes.
\end{abstract}

Keywords-Coverage Problem, Distance Relaying, Machine Learning, WSN

\section{Introduction}

Wireless Sensor Network (WSN) comprises battery-powered sensor devices called "nodes" that reply to homogeneous neighbors for routing and transmissions. The data exchange between the neighbors is performed using wireless links that define a node's connectivity [1]. In the neighbor discovery process, either reactive or proactive routing protocols are employed for identifying an active link. An active link is identified based on the neighbor's response within the maximum time-out interval to the sender. Additionally, the neighbor must be present within the communication range of the transmitting node [2]. The routing protocols exploit these features in routing, node displacement, or repositioning. The density of the nodes determines the active links and so the connectivity between them. Battery energy is an influencing factor for link lifetime and hence the transmissions. Energy conserved and optimally utilizing nodes is said to have enduring connectivity in single and multiple hops [3].

The node's communication range is subject to change depending on its energy and mobility. However, the node placement must ensure maximum network coverage, preventing holes [4]. A coverage hole is created if a node or group of nodes are absent from a network spot due to energy drain or mobility. Then this region experiences no connectivity regardless of the high node coverage. This problem interrupts neighbor discovery resulting in dead-end routing and lossy transmissions [5]. Precise node deployment, relay-based connectivity, energy optimization, location-aware routing are some techniques that address the coverage-hole problem. Besides, node redundancy and blind spots also result in a coverage-hole problem, and hence Coverage and Connectivity (CC) solutions are introduced [6]. Energy is again an influencing factor in determining the transmit antenna's coverage and however, if energy optimization is undergone, then desired node coverage is less feasible to be achieved. Therefore, a proposed solution must balance the energy, coverage, and connectivity issues in a WSN for efficient data relaying [7]. The proposed method relies on differential metrics for surpassing the coverage hole problem. Routing coupled hole mitigation and route discovery are balanced between different sensor nodes. Besides, the intervals are sufficiently allocated regardless of the paused transmissions, in achieving less loss. The article's organization is presented as: Section 2 discusses the works related to the coverage hole problem and its 
mitigation methods introduced by several authors in the past. In Section 3, the proposed relaying method with the identified problem and solution is presented. The following Section 4 discusses the comparative analysis using different metrics and methods and Section 5 concludes the article with the experimental findings.

\section{Related Work}

Khalifa et al. [8] proposed Heuristic Hole Healing Algorithm (H3A) for coverage gap mitigation. This algorithm relies on the flexible node sensing range based on their dynamic position for mitigating coverage gaps. Neighbor selection is performed using the remaining energy and coverage intersection for maximizing network lifetime.

A distributed coverage-hole mitigation approach using machine learning is presented by Hajjej et al. [9]. Modified node distribution and its sensing range are accounted for pursuing game theory for addressing the afore-mentioned issue. The learning process is responsible for node repositioning recommendations for reducing energy consumption.

Sun et al. [10] introduced connectivity and coverage algorithm for improving the network lifetime in a hole influenced scenario. This algorithm identifies node replications and mitigates blind coverage. Post this mitigation, and the shortest path is employed in the blind coverage for improving the connectivity.

Improved and cooperative particle swarm optimization-based node deployment method is discussed in [11]. The proposed method addresses target coverage issues in WSNs, using fuzzy logic. This method is reliable in improving the network lifetime regardless of the node density.

Zakariayi and Babaie [12] proposed a clustering algorithm for maximizing region coverage in WSNs. The coverage is performed in a hexagonal structure of connecting nodes by differentiating static and mobile nodes. In this process, energy and neighbor density are cluster determining factors for improving network and coverage span.

Improved Artificial Bee Colony (IABC) algorithm is projected by Yue et al. [13] in addressing the coverage and connectivity problem of WSNs. In this algorithm, a free search model using pheromone sensitivity is used for mitigating convergence. This algorithm effectively improves network efficiency, coverage, and connectivity by reducing data replications and network traffic.

Torshizi and Sheikhzadeh [14] introduced an optimal coverage algorithm for WSNs. This algorithm identifies and removes replicated nodes causing blind spots. In this process, cellular automata are used for disabling redundancies. The proposed method improves the network coverage, conserves energy and network lifetime.

\section{Distance-to-Coverage Transformed Relaying}

The proposed method aims to reduce the transmission loss due to coverage-hole problems in WSNs. A coverage hole in the wireless network occurred due to the absence of routed neighbors and failed route. This is frequent as the nodes pass limited energy and lack proper conservation. Based on this factor, the problem is defined as follows:

\subsection{Problem Definition:}

Let $\{1,2, \ldots, n\} \in N$ denotes a set of sensor nodes dispersed in a $X * Y$ dimension region. Let $t_{n}$ denote the transmitting range of a node, then

$$
\forall n, \rho(L \mid n)=\left\{\begin{array}{l}
n^{\prime}, \text { if } d(n, n+1) \leq t_{n} \\
0, \text { if } d(n, n+1)>t_{n}
\end{array}\right.
$$

In eq. (1) the variable $\rho(L \mid n)$ refers to the probability of a link (L) with its neighbor if $\rho(L \mid n)=0$, then the neighbor is unavailable within the distance $(d)$. However, the $n$ is not constant and hence, the transmitting neighbors $\left(N_{T}\right)$ is computed as

$$
N_{T}=\frac{\rho(L \mid n)}{n} *\left[1-\frac{\rho(L \mid n)}{N}\right]
$$

In the above eq., the actual neighbor during transmission is estimated. The actual problem (coverage) exists, if $N_{T}<($ or $) \ll[\rho(L \mid n) * n]$ in any $\left(d_{T}-d_{D}\right)$ interval. The variables $d_{T}$ and $d_{D}$ are used to represent data transmitting and delivery delay, respectively. Now, the aim is to maximize the coverage ration regardless $N_{T}$ such that 


$$
\begin{aligned}
& f[K=N * \rho(L \mid n)]=\left\{\begin{array}{c}
t_{n}\left(1-\frac{t_{n}}{N_{T}}\right)^{n-1}, n \in\{N\} \forall N_{T}=\rho(L \mid n) * n \\
\left(1-\frac{t_{n}}{N}\right)^{n-1}, n \in\left\{N_{T}\right\} \forall N_{T}<\rho(L \mid n) * n
\end{array}\right\} \\
& \text { Valid for } \theta=\frac{\sum_{p} \rho(L \mid n)}{X * Y}, p \in[X * Y]=\max \left\{\frac{\rho(L \mid n)}{1}, \frac{\rho(L \mid n)}{2}, \ldots, \frac{\rho(L \mid n)}{N_{T}}\right\}
\end{aligned}
$$

In eq. (3), the variable $\theta$ is the coverage rate, and $p$ is any position of $n$ in $X * Y$ dimension. The distribution function $f($. ) Computed for $k$ transmission of a node $n$. Therefore, the distribution based on $\rho(L \mid n)$ must be capable of achieving/ balancing $\theta=\max \left\{\frac{\rho(L \mid n)}{N_{T}}\right\}$.

\subsection{Relaying Process}

A conventional routing process relies on the distance factor for data transmission. The further routing changes are forecasted based on residual energy assumptions. The communication intervals are computed at the end of the transmission process. In this proposed method, the time intervals are adaptive based on the energy-to-path existence factor. This prevents intervals-less data losses, wherein delay remains uncompromised. The conclusive machine learning used in this proposed method eyes on the $\rho(L \mid n)$ and $f($.$) for retaining transmission paths to$ the sink node. The computations for energy to path factor $(\tau)$ are given as follows: The residual energy of a node $\left(E_{r}\right)$ is computed as $E_{r}=E_{o}-E_{c}$, where $E_{o}$ and $E_{c}$ are the initial and consumed energies of a node.

$$
\left.\begin{array}{c}
\rho\left(\sum_{i=1}^{n} i\right)=\left\{[\rho(L \mid n) * n]-\frac{\theta}{N}\right\}^{n} \\
\text { such that } \\
\tau=\sum_{j=1}^{k}\left\{\left(E_{r}\right)_{j}\left[1-\rho\left(\sum_{i=1}^{n} i\right)\right]\right\}^{N-1} \\
\text { for all } \\
\tau * \theta=\frac{1-\left[1-\rho(L \mid n)^{N}\right]}{N_{T}} \\
\text { and hence, } \\
\tau=\frac{1-\left[1-\rho(L \mid n)^{N}\right]}{N_{T}} * \frac{(X * Y)}{\sum_{p} \rho(L \mid n)}
\end{array}\right\}
$$

In eq. (4), the probability of a single $n$ available for transmission is computed first. If this is valid, then the energyto-path factor is computed, achievable for $\tau$ as substituted for $\theta$ from eq. (3). The imbalance occurs if $f($ ), given in eq. (3) does not achieve $\theta$ for $\tau$ estimated in Eq. (4). In eq. (4), the chances for high and utmost possible $\tau$ in the presence (all $n \in N$ ) and absence $\left[N_{T}<\rho(L \mid n)\right]$ of the nodes is given. The conclusive machine learning verifies the above conditions for determining the further relaying intervals. The learning process is performed in two different cases (i.e.) for $\tau$ changes and path-existence. In both cases, the transmission intervals are adapted for loss-less performance. The cases are explained below:

\section{Case 1: Energy-to-Path Existence Analysis}

This case is analyzed for identifying the node-level setback in $N_{T}$ identification. In this analysis, eq. (1) is validated for the $\theta$ estimated in Eq. (3). As mentioned, the $\max \left\{t_{n}\right\} \forall n \in N_{T}$ is the minimum analyzable criteria for relaying. This analyzing problem is illustrated in Fig 1 . Series.

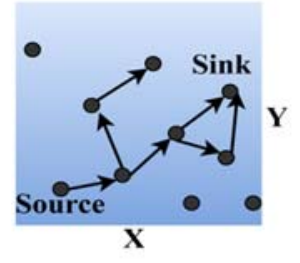

(a) Conventional Scenario

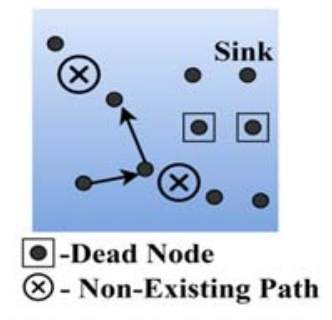

(b) Dead Node Scenario

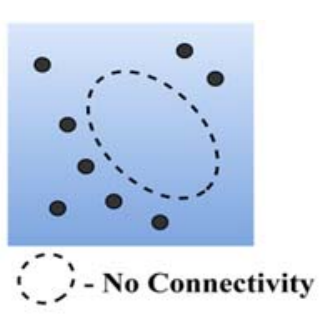

(c) Connection Less Region

Fig. 1.Energy-to-Path Existence Illustration

The conventional scenario loses its connectivity due to dead nodes and non-existing paths. This problem is handled by analyzing $\tau$ for maximizing $\theta$. Let $I$ be the actual interval for data transmission between the sources and sink nodes. This interval is achievable in Fig.1 (a) scenario, whereas the rest of the cases does not achieve this. Therefore, the occurring connection less scenario is inevitable for which $N_{T}$ analysis is performed. The learning 
sequence is first instigated for $I$ iterates; the interrupt in data relaying converges the iterations. The convergence $(\Delta)$ at which the iteration halts is estimated in Eq. (5)

$$
\left.\Delta=\frac{\rho(L \mid n) * n * d_{D}}{\rho\left(\sum_{i=1}^{n} i\right) * d_{T}}\right\}
$$

Based on this $\Delta$, the learning process is instigated for case $1-\mathrm{a}$ formal representation of the learning process presented in Fig. 2.

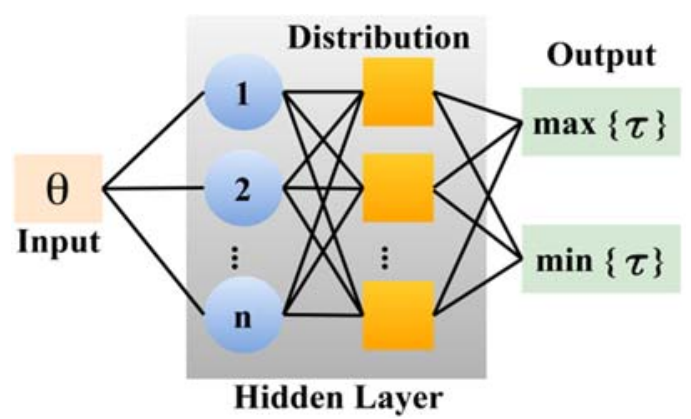

Fig. 2.Learning Process for $\boldsymbol{\tau}$ Assessment

The hidden layer processes are validated for 1 to $(I-\Delta)$ intervals of the $n$. This is computed as

$$
\left.\begin{array}{c}
h_{1}=\tau_{1} \\
h_{2}=\frac{\tau_{2}-\rho(L \mid n)_{1}}{\left(N_{T}\right)_{2}} \\
\vdots \\
h_{I-\Delta}=\tau_{I-\Delta}-\frac{\rho(L \mid n)_{I-\Delta-1}}{\left(N_{T}\right)_{I-\Delta}}
\end{array}\right\}
$$

The output of the hidden layer process identifiesmax $\left\{h_{1}, h_{2}, \ldots, h_{I-\Delta}\right\}$ andmin $\left\{h_{1}, h_{2}, \ldots, h_{I-\Delta}\right\}$. Therefore, the node with $\max \{\tau\}$ is the first and updated $(1$ to $I-\Delta) \theta$. The pursuing relaying process adapts to $(I-\Delta)$ interval in selectingmax $(\tau)$ such that $\left[N_{T}=\rho(L \mid n) * n\right]$ is satisfied. The $h$ instance where the above condition fails is the convergence, and hence a new interval is used for relaying. It is to be noted that $E_{r}$ has a direct impact over $\Delta$ and, therefore, $\max \left\{h_{1} \cap h_{2}, h_{2} \cap h_{3}, \ldots, h_{I-\Delta-1} \cap h_{I-\Delta}\right\}$ is the next possible nodes for multipath in the relaying.

\section{Case 2: Transformed Relaying}

As mentioned earlier, $E_{r}$ impacts $\Delta$ and hence $\tau$ wherein the coverage is observed as a flow. This case identifies maximum coverage nodes for preventing early convergence due to energy drain. Therefore, $f($.$) is analyzed to$ retaining the transformed relaying. Here, the interval convergence is aimed to be improved to $I$ from $(I-\Delta)$. This relaying method is checked for its balancing between path existence and intervals, reducing the information loss. First, the minimum and maximum $\theta$ is estimated from $f($.$) in any (I-\Delta)$ is computed. The computations are given below.

$$
\theta=\left\{\begin{array}{c}
\frac{\sum_{p} \rho(L \mid n)}{\rho\left(\sum i\right) \forall i \in N_{T}} \text { is maximum, if } n \in N=N_{T} \\
\frac{1}{\tau * N_{T}} n * \rho(L \mid n), \text { is minimum, if } N_{T} \ll[\rho(L \mid n) * n]
\end{array}\right.
$$

The actual $\theta$ estimated in Eq. (3) is balanced for the above to verify if $f[k=n * \rho(L(n)]$ holds in $(I-\Delta)$. Based on the balancing,

$$
\left.\begin{array}{c}
\rho\left(\sum_{i=1}^{n} i\right)=X * Y \forall n \in N=N_{T}[\text { Subs. for eqs. (7) and (3) }] \\
\text { and } \\
n=\frac{N_{T^{*} \tau}}{X * Y} \forall N_{T} \ll[\rho(L(n) * n)]
\end{array}\right\}
$$

From eq. (8), the first case is valid for $f($.$) , whereas the distribution for the second case requires transformation.$ It is based onmax $\{\tau\}$ as computed in case 1 . Therefore, the learning process is reversed with training for verifying $f($.$) in the entire (I-\Delta)$ instance. This identifies new $n$ for data relaying without loss and early convergence. The output of the learning process is used for modifying the distribution such that $\theta$ is retained just greater than the minimum $\tau$. Fig. 3 illustrates the learning process for verifying $f($.$) .$ 


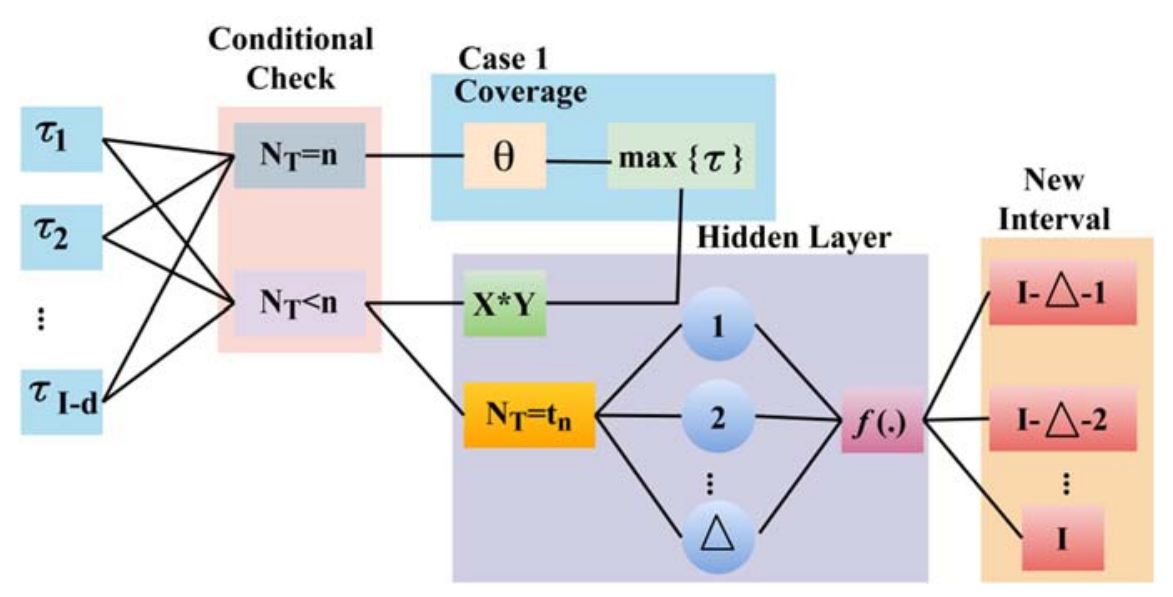

Fig.3.Learning Process for $\boldsymbol{\theta}$ Verification

This hidden layer process performs case 1 classification covering $(X * Y)$ with the initial distribution. Contrarily, the second process for the condition $N_{T}<n$ is given as follows

$$
\left.\begin{array}{c}
h_{I-\Delta}=\frac{f(.)_{I-\Delta}-\rho(L \mid n)_{I-\Delta-1}}{N} \Rightarrow f(.)_{I-\Delta}=t_{n}\left(1-\frac{t_{n}}{N-N_{T}}\right)^{I-\Delta} \\
h_{I-\Delta-1}=\frac{f(.)_{I-\Delta-1}-\rho(L \mid n)_{I-\Delta-2}}{N} \Rightarrow f(.)_{I-\Delta-1}=t_{n}\left(1-\frac{t_{n}}{N-N_{T}-\rho(L \mid n) * n}\right)^{I-\Delta-1} \\
\vdots \\
h_{I}=f(.)_{I}-\frac{\rho(L \mid n)_{I-1}}{N} \Rightarrow f(.)_{I}=t_{n}\left(1-\frac{t_{n}}{N}\right)^{I}
\end{array}\right\}
$$

In eq. (9), the modified distribution for pursuing data transmission in $(I-\Delta, I)$ interval is estimated. The hidden layer process other than case 1 is performed for this Assessment. This validation is pursued until a max $\left\{t_{n}\right\}$ node is identified. Post this identification; the distribution is modified for non-intervening transmissions.

The changes are updated to the routing paths for maximum coverage. Hence both cases are keen on mitigating connectivity and coverage issues in handling holes in transmission. It is achieved by $\max \left\{h_{1} \cap h_{2}, \ldots h_{I-\Delta-1} \cap\right.$ $\left.h_{I-\Delta}\right\}$ for connectivity and modified $f(.) \forall N_{T} \ll[\rho(L \mid n) * n]$ condition.

\section{Results and Discussion}

The proposed method's performance is analyzed using experiments performed using the network simulator version 2 software. This simulation is carried out in a Linux operating system in which the network region is generated for $750 \mathrm{~m} \times 500 \mathrm{~m}$ in which 50 sensor nodes are placed. The simulation is executed for $140 \mathrm{~s}$ using dynamic source routing protocol. The nodes are capable of covering $25 \mathrm{~m}$ range, equipped with an $E_{o}$ of $20 \mathrm{~J}$. With this setup, the metrics coverage factor, data loss \%, and energy consumption are compared. In the comparison section, the existing $\mathrm{H} 3 \mathrm{~A}[8]$ and IABC [13] methods are accounted.

\subsection{Coverage Factor}
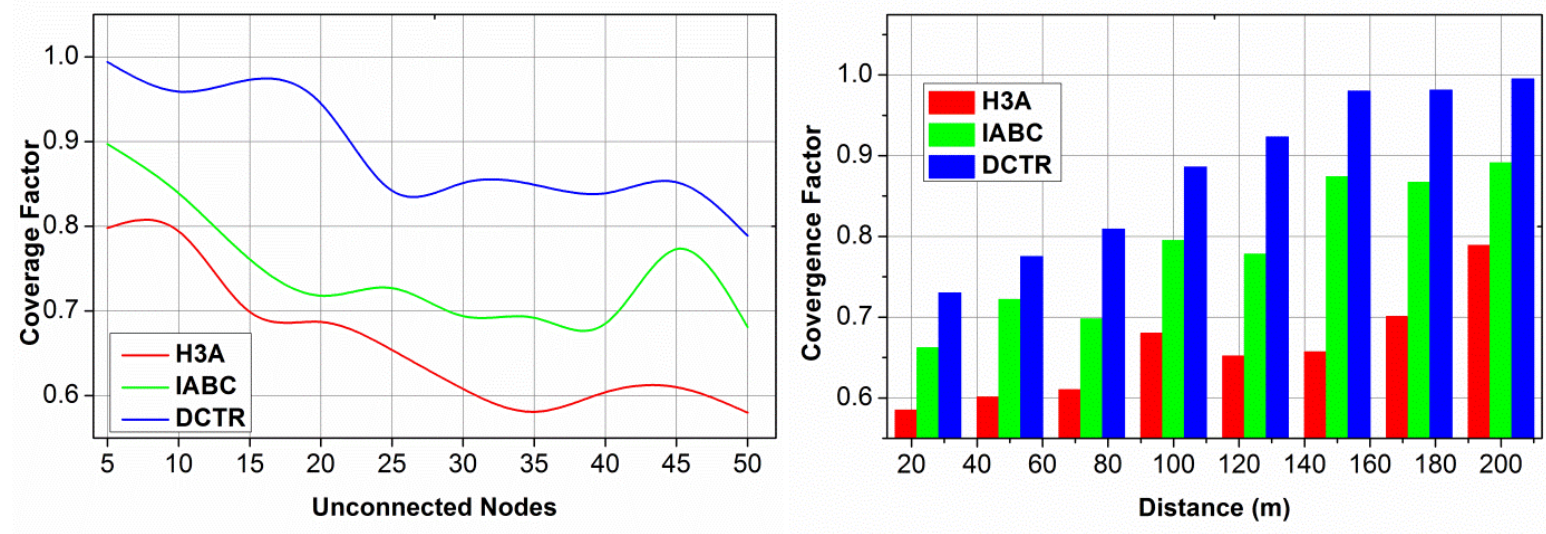

Fig. 4 Coverage Factor for Unconnected Nodes and Distance (m) 
Fig. 4 presents the comparative analysis of coverage factors for different unconnected nodes and distances. The proposed method handles connectivity and coverage independently based on $\max \{\tau\}$ and $f($.$) . The precise n$ selection and distribution are recommended by the learning process for maximized coverage. In this process, the $\tau$ based learning identifies all possible chance for satisfying $N_{T}=[\rho(L \mid n) * n]$. Besides, the interval-based $f($. improves the connectivity for maximizing $\theta$. Therefore, both the cases perform mutual Assessment in neighbor route filling and distance coverage.

\subsection{Data Loss}
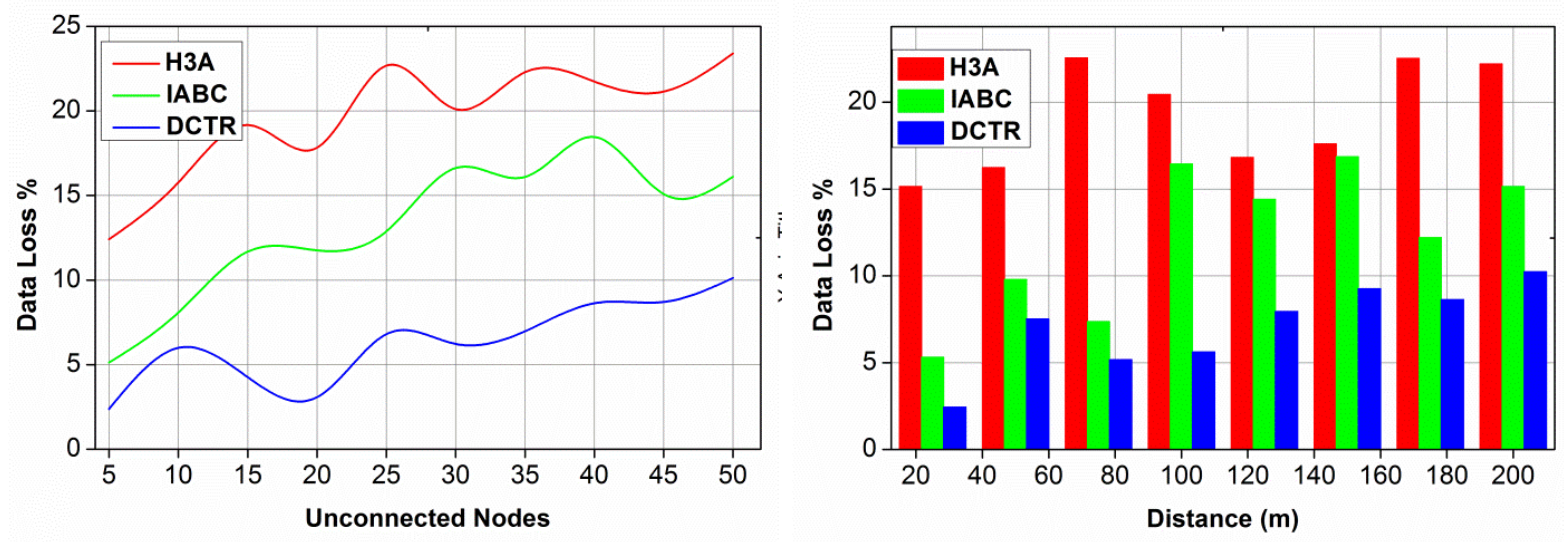

Fig. 5 Data Loss \% for Unconnected Nodes and Distance (m)

The proposed method shows less data loss for unconnected nodes and distance (Refer to Fig. 5). The convergence due to early energy drain is analyzed using $\tau$ factor. This initial Assessment determines $\Delta$ deciding the need for alternate $n$ or $f($.$) . However, this convergence is deciding the need for alternate n$ or $f($.$) . However, this$ convergence is addressed in case 2 by validating $N_{T} \leq n$ condition. The learning recommends the $\Delta$ for $\max \{\tau)$ such that $f(.)_{I}$ presents a new distribution for increasing $(I-\Delta)$ to $I$. Such identified $n$ satisfying the condition in Eq. (3) and (8) are used for relaying. These selected nodes prolong the transmission time, reducing data loss.

\subsection{Energy Consumption}
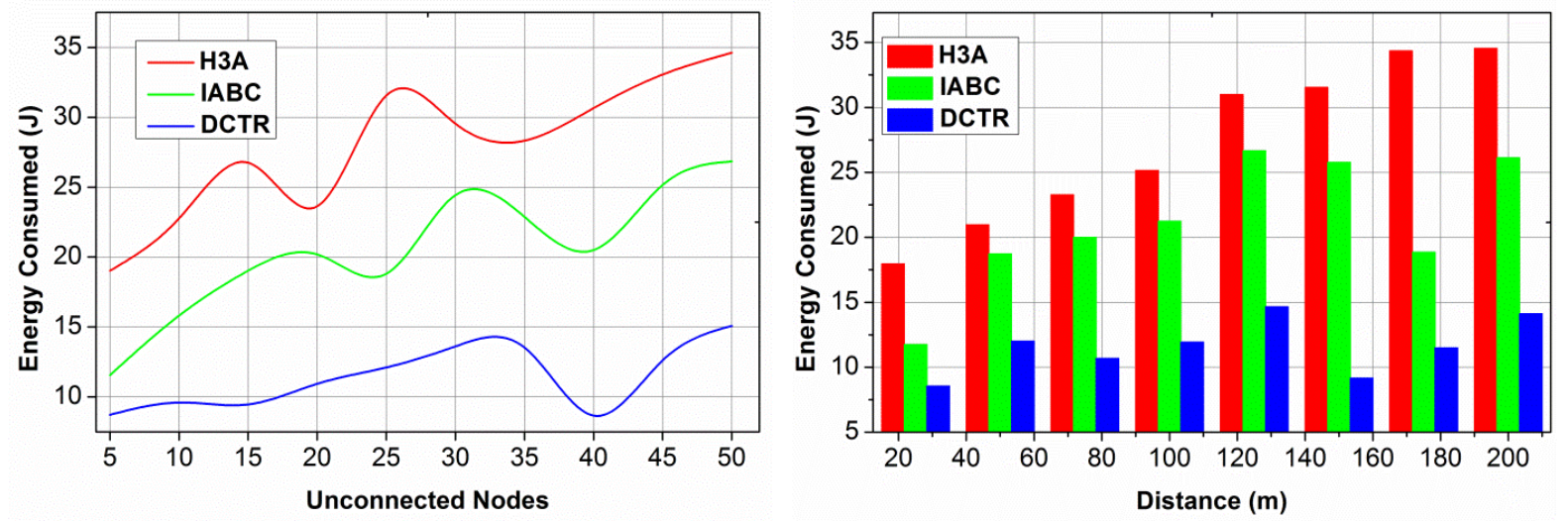

Fig. 6 Energy Consumption for Unconnected Nodes and Distance (m)

The impact of $E_{r}$ in $\tau$ and $\theta$ are considered in this proposed method for improving the data transmissions. However, the impact of $E_{r}$ is first identified using $\tau$ for whichmax $\left\{h_{1} \cap h_{2}, h_{2} \cap h_{3}, \ldots, h_{I-\Delta-1} \cap h_{I-\Delta}\right\}$ is estimated. Therefore, the energy utilization is confined within the $I-\Delta$ interval without retransmission. In the second case, re-organized $f($.$) ensures confined energy consumption other than periodic neighbor switching.$ Hence, the overall energy consumed in $[1, I-\Delta]$ and $[I-\Delta, I]$ is the same as that of a confined transmission. Here, the retransmission effects are less due to minimum path assurance, by mitigating $N_{T} \ll[\rho(L \mid n) * n]$ condition. The comparative analysis results are tabulated in Tables 1 and 2 with the inference. This analysis results are tabulated in concern to $\mathrm{H} 3 \mathrm{~A}$ and IABC independently. Finally, the mean of the proposed method with $\mathrm{H} 3 \mathrm{~A}$ and IABC is accounted as the concluding achievement. 


\begin{tabular}{|l|l|l|l|}
\hline Metric & H3A & IABC & DCTR \\
\hline Coverage Factor & 0.58 & 0.681 & 0.789 \\
\hline Data Loss (\%) & 23.4 & 16.11 & 10.117 \\
\hline Energy Consumed (J) & 34.61 & 26.84 & 15.083 \\
\hline
\end{tabular}

Table 1. Comparative Analysis Results for Unconnected Nodes

The proposed method improves the coverage factor by $7.93 \%$, reduces data loss by $9.64 \%$, and energy consumed by $8.49 \%$.

\begin{tabular}{|l|l|l|l|}
\hline Metric & H3A & IABC & DCTR \\
\hline Coverage Factor & 0.789 & 0.891 & 0.995 \\
\hline Data Loss (\%) & 22.2 & 15.15 & 10.233 \\
\hline Energy Consumed (J) & 34.54 & 26.12 & 14.126 \\
\hline
\end{tabular}

Table 2.Comparative Analysis Results for Distance (m)

The proposed DCTR improves coverage factor by $7.75 \%$ for the different distances, reducing data loss and energy consumed by $8.4 \%$ and $8.9 \%$, respectively.

\section{Conclusion}

For addressing the coverage and connectivity issues in wireless sensor networks, this paper introduced Distanceto-Coverage Transformed Relaying (DCTR) method. It is seen that the discovery and relaying balances energy and neighbor availability for prolonged path availability. The learning process identifies the coverage rate and packet distribution factor for identifying reliable nodes. Based on the learning recommendations, the neighbors/ nodes are selected for transmission. Therefore, the neighbor availability is high, reducing the wait time between the intervals and hence improving the connectivity. From the experimental analysis, the proposed method is seen to improve the coverage rate by $7.93 \%$, reduces data loss by $9.64 \%$, and energy consumed by $8.49 \%$ for different unconnected nodes.

\section{References}

[1] Ammari, H. M. (2021). Connected k-coverage in two-dimensional wireless sensor networks using hexagonal slicing and area stretching. Journal of Parallel and Distributed Computing.

[2] Chen, D. R., Chen, L. C., Chen, M. Y., \& Hsu, M. Y. (2019). A coverage-aware and energy-efficient protocol for the distributed wireless sensor networks. Computer Communications, 137, 15-31.

[3] Elhabyan, R., Shi, W., \& St-Hilaire, M. (2019). Coverage protocols for wireless sensor networks: Review and future directions. Journal of Communications and Networks, 21(1), 45-60.

[4] Farsi, M., Elhosseini, M. A., Badawy, M., Ali, H. A., \& Eldin, H. Z. (2019). Deployment techniques in wireless sensor networks, coverage and connectivity: A survey. IEEE Access, 7, 28940-28954.

[5] Hajjej, F., Hamdi, M., Ejbali, R., \& Zaied, M. (2020). A distributed coverage hole recovery approach based on reinforcement learning for Wireless Sensor Networks. Ad Hoc Networks, 101, 102082.

[6] Khalifa, B., Khedr, A. M., \& Al Aghbari, Z. (2019). A coverage maintenance algorithm for mobile WSNs with adjustable sensing range. IEEE Sensors Journal, 20(3), 1582-1591.

[7] Nguyen, T. N., Liu, B. H., \& Wang, S. Y. (2019). On new approaches of maximum weighted target coverage and sensor connectivity: Hardness and approximation. IEEE Transactions on Network Science and Engineering, 7(3), 1736-1751.

[8] Qasim, T., Zia, M., Minhas, Q. A., Bhatti, N., Saleem, K., Qasim, T., \& Mahmood, H. (2018). An ant colony optimization based approach for minimum cost coverage on 3-D grid in wireless sensor networks. IEEE Communications Letters, 22(6), 1140-1143.

[9] Sun, G., Liu, Y., Li, H., Wang, A., Liang, S., \& Zhang, Y. (2018). A novel connectivity and coverage algorithm based on shortest path for wireless sensor networks. Computers \& Electrical Engineering, 71, 1025-1039.

[10] Torshizi, M., \& Sheikhzadeh, M. J. (2020). Optimum K-coverage in wireless sensor network with no redundant node by cellular learning automata. Wireless Personal Communications, 110(2), 545-562.

[11] Vatankhah, A., \& Babaie, S. (2018). An optimized bidding-based coverage improvement algorithm for hybrid wireless sensor networks. Computers \& Electrical Engineering, 65, 1-17.

[12] Yarinezhad, R., \& Hashemi, S. N. (2020). A sensor deployment approach for target coverage problem in wireless sensor networks. Journal of Ambient Intelligence and Humanized Computing, 1-16.

[13] Yue, Y., Cao, L., \& Luo, Z. (2019). Hybrid artificial bee colony algorithm for improving the coverage and connectivity of wireless sensor networks. Wireless Personal Communications, 108(3), 1719-1732.

[14] Zakariayi, S., \& Babaie, S. (2019). DEHCIC: a distributed energy-aware hexagon based clustering algorithm to improve coverage in wireless sensor networks. Peer-to-Peer Networking and Applications, 12(4), 689-704. 


\section{Authors Profile}

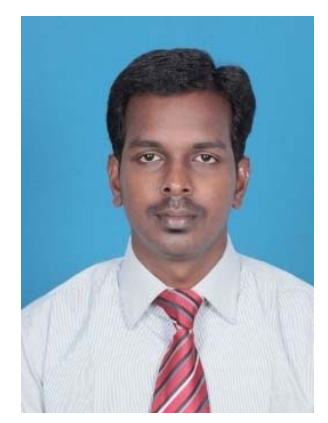

R.Rajesh, working as Associate Professor in Department of computer Science and Engineering, IFET College of Engineering, Villupuram. He received his master degree in Information Technology in Sathyabama University. He has published eight research papers in reputed International Journals and ten International conferences. He received funds for organizing Seminar and conference from ISRO and DHR. He acted as one of the speakers and organized various Seminars and Workshop. His main research work focuses on Inter of Things and Wireless Sensor Networks. He has 9 years of teaching experience and he has guided more than 10 Under graduations and Post graduations.

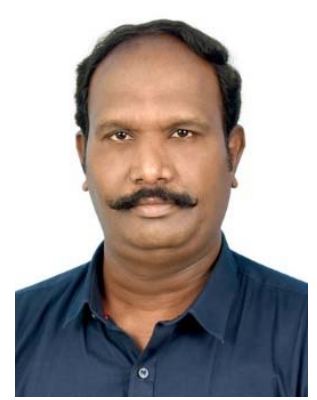

Dr.V.Tamizhazhagan, working as Assistant Professor in Department of Information Technology, Annamalai University, Chidambaram. He received his Ph.D degree in Information Technology in Annamalai University. He has published fourteen research papers in reputed International Journals and ten International conferences and it's also available online. He attended \& organized various workshops. His main research work focuses on Data Mining, Network Security, Mobile Computing and Computer Networks. He has 17 years of teaching experience. He guided 10 Post graduation, many under graduation projects and guiding 4 Ph.Ds. 